\title{
Estudo de influência de modelos difusivo e advectivo para a dispersão de poluentes no Rio Paraibuna
}

Incidentes envolvendo despejo de poluentes em meios hídricos vem se tornando cada vez mais frequentes, tornando importante o estudo de novos métodos a fim de desenvolver estratégias que permitam prever esses episódios e amenizar seus danos à natureza e à vida humana. O presente trabalho tem como objetivo fazer uma comparação entre modelos unidimensionais de difusão e advecção para a dispersão de poluentes usando como exemplo de aplicação o rio Paraibuna, com o propósito de discutir e explorar as características de cada modelo. Para isso, foram utilizados o método GILTT (Generalized Integral Laplace Transform Technique) e o método de Taylor para obter a solução do modelo difusivo e o método de Separação de Variáveis com Transformada de Laplace para a solução do modelo advectivo. São apresentadas simulações de dispersão de poluentes para cada metodologia e os resultados são discutidos e comparados com dados reais observados obtidos em literatura. Os resultados mostram que no modelo advectivo, a nuvem de poluente permanece compacta durante todo o processo, apresentando uma boa aproximação do momento no qual ocorre a concentração máxima em uma posição fixa; enquanto no modelo difusivo, para ambas as soluções, nota-se que a quantidade de poluente é maior em locais próximos a fonte, levando um tempo considerável para sua diluição. Foi possível concluir que cada modelo fornece informações satisfatórias que vão de encontro as expectativas teóricas para a dispersão de poluentes e que a metodologia utilizada foi prática e eficaz para os propósitos deste trabalho.

Palavras-chave: Dispersão de poluentes; Advecção-Difusão; Transformada de Laplace; GILTT; Rio Paraibuna.

\section{Study of influence of diffusive and advective models for the dispersion of pollutants on River Paraibuna}

\begin{abstract}
Incidents involving dumping of pollutants into water are becoming more and more frequent, making it important to study new methods in order to develop strategies to predict these episodes and mitigate their damage to nature and human life. The present work aims to make a comparison between one-dimensional models of diffusion and advection for a dispersion of pollutants using the Paraibuna river as an application example, with the purpose of discussing and exploring the characteristics of each model. For this purpose, it was used the GILTT (Generalized Integral Laplace Transform Technique) method and the Taylor method in order to solve the diffusive model and the Separation of Variables method with Laplace Transform for solving the advective model. Pollutant dispersion simulations are presented for each methodology and the results are discussed and compared with actual observed data obtained in the literature. Results show that in the advective model, the pollutant wave remains compacted throughout the whole process, revealing a good approximation of the moment at which occurs the highest concentration in a fixed position; on the other hand, in the diffusive model, it is noticeable that in both approaches the amount of pollutant is higher at locals near the source of injection, taking some noticeable time for its dilution, revealing how the molecular diffusion process occurs inside the pollutant wave. It was possible to conclude that each model provides satisfactory information that meets the theoretical expectations for the dispersion of pollutants and that the methodology used was practical and effective for the purposes of this work.
\end{abstract}

Keywords: Pollutant dispersion; Diffusion-Advection; Laplace Transform; GILTT; Paraibuna River.

Igor da Cunha Furtado

Instituto Federal Sul-Rio-Grandense, Brasil http://lattes.cnpq.br/9625380341591716 http://orcid.org/0000-0002-0776-3225 igorjara@gmail.com

\section{André Rickes (iD)}

Universidade Federal de Pelotas, Brasil http://lattes.cnpq.br/4200293194324021 http://orcid.org/0000-0002-9254-0199 andre.rickes@hotmail.com

Tamires Fonseca de Almeida (iD) Universidade Federal de Pelotas, Brasil http://lattes.cnpq.br/9171847615534939 http://orcid.org/0000-0003-1384-7828 tamiresfonsecadealmeida@hotmail.com

DOI: 10.6008/CBPC2179-6858.2021.001.0022

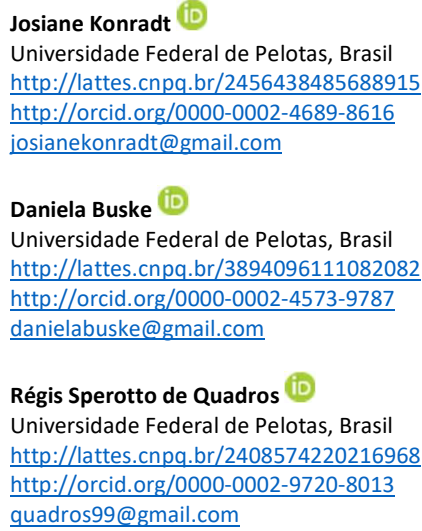

Universidade Federal de Pelotas, Brasil http://lattes.cnpq.br/3894096111082082 http://orcid.org/0000-0002-4573-9787 danielabuske@gmail.com

Régis Sperotto de Quadros (iD)

Universidade Federal de Pelotas, Brasil

http://lattes.cnpq.br/2408574220216968

http://orcid.org/0000-0002-9720-8013

quadros99@gmail.com

\section{Guilherme Jahnecke Weymar in \\ Universidade Federal de Pelotas, Brasil http://lattes.cnpq.br/6324333547922986 http://orcid.org/0000-0001-8216-9122} guilhermejahnecke@gmail.com

\section{Referencing this:}

FURTADO, I.; RICKES, A.; ALMEIDA, T. F.; KONRADT, J.; BUSKE, D.; QUADROS, R. S.; WEYMAR, G. J.. Estudo de influência de modelos difusivo e advectivo para a dispersão de poluentes no Rio Paraibuna. Revista Ibero Americana de Ciências Ambientais, v.12, n.1, p.262275, 2021. DOI: http://doi.org/10.6008/CBPC2179- 


\section{INTRODUÇÃO}

A dispersão de poluentes em meios hídricos é um problema que vem ganhando cada vez mais destaque entre os órgãos de saúde e preservação da vida marinha. Ultimamente, nota-se que a industrialização sem planejamento e o desenvolvimento tecnológico e econômico da sociedade contribuem significativamente no aumento dos casos de descarte indevido de substâncias poluidoras em corpos hídricos (SOARES et al., 2016), dos quais pode-se exemplificar o despejo do esgoto de residências e comércios, ou ainda o despejo de efluentes tóxicos por indústrias. Os danos resultantes desses eventos podem ser minimizados através do conhecimento do processo de dispersão desses resíduos, e a modelagem matemática tem um papel importante na simulação de despejos de poluentes em rios, lagos e mares, criando soluções que preveem como se dá a dispersão desse poluente a partir do seu ponto e instante de derramamento (MAIA et al., 2001).

No estudo da dinâmica de poluentes inseridos em corpos hídricos, é necessário considerar dois processos importantes: a advecção e a difusão. Segundo Cremasco (2009), a advecção é o processo em que o soluto é carregado como consequência do movimento do meio, enquanto a difusão se dá pelas interações moleculares entre o soluto e o meio, espalhando-se da posição onde há maior concentração para a que há menor concentração do soluto. O processo de dispersão de poluentes acontece, sobretudo, como a combinação desses dois processos simultaneamente.

No presente trabalho, serão utilizados os métodos GILTT (BUSKE et al., 2017) e Taylor (SOARES et al., 2010) com o intuito de encontrar soluções analíticas para o modelo difusivo, e o método de Separação de Variáveis com Transformada de Laplace para determinar a solução do modelo advectivo. Ambos modelos utilizados para a dispersão de poluentes no rio Paraibuna, encontrado na bacia hidrográfica do rio Paraíba do Sul, na região sudeste do país, verificando a consistência de cada metodologia aplicada a um problema da natureza. Para isso, foram utilizados os parâmetros obtidos nas campanhas de campo realizados por Soares et al. (2010), aplicados na equação de difusão-advecção no modelo unidimensional.

A solução do problema pelo método GILTT constitui da construção de um problema auxiliar de SturmLiouville, expansão da concentração do poluente em uma série em termos das autofunções obtidas, substituição da expansão na equação a ser resolvida, e obtenção de uma equação diferencial ordinária na forma matricial, que por sua vez, é resolvida pela Transformada de Laplace (BUSKE et al., 2010). O método de Taylor, explorado no trabalho de (SOCOLOFSKY et al., 2002), resume-se em aplicar o teorema $\pi$ de Buckingham (ÇENGEL, 2007), reescrevendo a concentração por uma função genérica de uma variável adimensional, com propósito de transformar o problema em uma Equação Diferencial Ordinária (EDO) , e resolvê-la com o auxílio da Transformada de Fourier.

Já o método de Separação de Variáveis consiste em considerar a solução do problema como o produto de duas funções, cada uma dependendo de apenas uma variável independente, e substituir esse produto no problema a ser resolvido, obtendo duas EDO's (BOYCE et al., 2006). A partir disto, são encontradas as soluções das EDO's, que serão também funções de uma constante arbitrária. Reescrevendo 
a solução do problema original como produto das soluções das EDO's, utilizamos a Transformada de Laplace para determinar a constante arbitrária.

Como referência base, foram utilizados os trabalhos de Buske et al. (2011), Machado (2019), Moreira et al. (2009), Soares et al. (2010), Tirabassi et al. (2009), Vilhena et al. (2012) e Wortmann et al. (2005). Nesses estudos, o método GILTT foi usado com sucesso para a formulação da solução analítica para o problema da dispersão de poluentes.

\section{METODOLOGIA}

\section{Parâmetros hidrodinâmicos}

Os parâmetros hidrodinâmicos que serão adotados no presente trabalho, foram obtidos nas campanhas de campo de Soares et al. (2010), utilizando a injeção de traçadores no rio Paraibuna e analisando seu trajeto através do curso do rio. Segundo Bedmar (1972), pode-se denominar traçador como toda substância que permite estudar o comportamento do processo químico ou físico de um meio, quando incorporado a este. Bedmar (1972) também afirma que o traçador ideal para corpos hídricos deve ter comportamento idêntico ao da água e não produzir nenhuma reação quando incorporada a estes. Além disso, devem ser tomados cuidados essenciais na escolha do traçador ideal, que são: não utilizar substâncias tóxicas ou que, de alguma forma, possam prejudicar os seres vivos da região; priorizar substâncias que se encontram em baixas concentrações no local de estudo, para facilitar a análise dos traçadores; utilizar produtos de baixo custo; entre outros (BEDMAR, 1972). Com base nisso, no trabalho de Soares et al. (2010) foram utilizados traçadores fluorescentes Uranina, também conhecido como Fluoresceína Sódica (Acid Yellow 73, Color Index 45350) e Amidorodamina G Extra (Acid Red 52, Color Index 45220), utilizados na indústria têxtil e frequentemente empregados como traçadores de corpos hídricos (SOARES et al., 2010).

O ponto de localização da injeção dos traçadores se localiza no rio Paraibuna sob a ponte da BR 040 ( $\mathrm{km}$ 777), Distrito Industrial, e as equipes de monitoramento se posicionaram em seus postos para realizar a análise da passagem da nuvem de traçador por cada um dos postos tomados (SOARES et al., 2010). A Tabela 1 mostra a localização dos locais de injeção e de monitoramento dos traçadores:

Tabela 1: Síntese dos dados das seções de injeção e monitoramento.

\begin{tabular}{|l|l|l|l|}
\hline Seções & Coordenadas UTM & Distância Acumulada $(\mathrm{km})$ & Denominação \\
\hline S0 & $(661307 ; 7602540)$ & 0,0 & Injeção \\
\hline S1 & $(665622 ; 7597640)$ & 7,6 & Represa \\
\hline S2 & $(671189 ; 7593382)$ & 16,0 & Pontilhão \\
\hline S3 & $(673166 ; 7590816)$ & 20,0 & Posto Policial \\
\hline
\end{tabular}

Fonte: Avaliação da Capacidade de Transporte e Dispersão do Rio Paraibuna Utilizando Traçadores Fluorescentes. Trecho: Distrito Industrial a UHE Marmelos — Juiz de Fora/MG; Soares et al. (2010).

Tais postos de monitoramento foram escolhidos, principalmente, por conta da homogeneidade dos trechos monitorados, pela facilidade do acesso para a tomada das amostras e pela existência de uma seção de amostragem próxima ao ponto de injeção, para que sejam avaliados os parâmetros $D_{l}$ (coeficiente de dispersão longitudinal), $U$ (velocidade do rio) e a vazão do rio em cada posto de monitoramento (SOARES et al., 2010). Alguns dos dados observados foram reunidos na Tabela 2. 
Tabela 2: Comparação dos parâmetros hidrodinâmicos da campanha 1 - Amidorodamina G Extra.

\begin{tabular}{|l|l|l|l|l|l|}
\hline \multicolumn{2}{|l|}{ Traçador Amidorodamina G Extra } & Vertical 1 & $Q_{\text {calc }}\left(\mathrm{m}^{3} / \mathrm{s}\right)$ \\
\hline Seção & Local & Distância $(\mathrm{km})$ & $D_{l}\left(\mathrm{~m}^{2} / \mathrm{s}\right)$ & $U(\mathrm{~m} / \mathrm{s})$ & 15,66 \\
\hline 1 & Represa & 7,6 & 6,2 & 0,52 & 19,57 \\
\hline 2 & Pontilhão & 16,0 & 11,0 & 0,53 & 18,60 \\
\hline 3 & Posto Policial & 20,0 & 12,7 & 0,55 & 20,22 \\
\hline 4 & UHE Marmelos & 27,0 & 16,0 & 0,53 & \\
\hline
\end{tabular}

Fonte: Avaliação da Capacidade de Transporte e Dispersão do Rio Paraibuna Utilizando Traçadores Fluorescentes. Trecho: Distrito Industrial a UHE Marmelos — Juiz de Fora/MG; Soares et al. (2010).

\section{Equação de Difusão-Advecção}

No estudo da dispersão de poluentes, existem alguns conceitos fundamentais que descrevem os fenômenos físicos de um soluto transportado em meios hídricos, atmosféricos, entre outros. No caso hidrodinâmico, a equação que descreve o fenômeno da dispersão de poluentes é obtida baseando-se no princípio da conservação de massa (equação da continuidade). Considerando $C$ a concentração de uma espécie genérica que se conserva em meios aquáticos, tem-se:

$\frac{\partial C}{\partial t}+U \frac{\partial C}{\partial x}+V \frac{\partial C}{\partial y}+W \frac{\partial C}{\partial z}+S=0$,

onde $C$ é a concentração do poluente $\left(\mathrm{mg} / \mathrm{m}^{3}\right)$, $t$ é o tempo após a injeção $(\mathrm{s}), x, y$ e $z$ representam as coordenadas retangulares da localização $(m), U, V$ e $W$ representam as componentes da velocidade instantânea do escoamento nas direções $x, y$ e $z(\mathrm{~m} / \mathrm{s})$, respectivamente, e $S$ denota o termo fonte. Considera-se o termo fonte quando a concentração analisada sofre transformações, por processos físicos, químicos ou biológicos. Quando a concentração do poluente não sofre nenhum processo desse tipo, ocorre apenas os processos de difusão e advecção e o termo S é desconsiderado (MACHADO, 2019).

A partir disto, de acordo com a decomposição de Reynolds, uma variável como a velocidade direcionada e a concentração, que são consideradas turbulentas, pode ser decomposta em um valor médio e uma flutuação em torno deste valor médio (TENNEKES et al., 1983). Logo, aplica-se na equação (1) as decomposições:

$$
U=\bar{U}+U^{\prime} ; \quad V=\bar{V}+V^{\prime} ; \quad W=\bar{W}+W^{\prime} ; \quad C=\bar{C}+C^{\prime} ;
$$

Onde cada termo denotado por uma barra superior representa seu valor médio, e cada termo representado por uma linha representa sua flutuação.

Além disso, é utilizada a lei de Fick, que afirma que, no processo de uniformizar a concentração no corpo hídrico, o fluxo turbulento da concentração analisada é proporcional ao gradiente de concentração média, mas possui sentido oposto. Mais precisamente, escreve-se da forma:

$$
\overline{U^{\prime} C^{\prime}}=-\varepsilon_{x} \frac{\partial \bar{C}}{\partial x} ; \quad \overline{V^{\prime} C^{\prime}}=-\varepsilon_{y} \frac{\partial \bar{C}}{\partial y} ; \quad \overline{W^{\prime} C^{\prime}}=-\varepsilon_{z} \frac{\partial \bar{C}}{\partial z}
$$

onde $\varepsilon_{x}, \varepsilon_{y}$ e $\varepsilon_{z}$ são os coeficientes de difusão turbulenta nas direções $x, y$ e $z$ respectivamente $\left(\mathrm{m}^{2} / \mathrm{s}\right)$. Aplicando os procedimentos anteriores na equação (1), após manipulações matemáticas, é encontrada

$$
\frac{\partial \bar{C}}{\partial t}+\bar{U} \frac{\partial \bar{C}}{\partial x}+\bar{V} \frac{\partial \bar{C}}{\partial y}+\bar{W} \frac{\partial \bar{C}}{\partial z}=\frac{\partial}{\partial x}\left(\varepsilon_{x} \frac{\partial \bar{C}}{\partial x}\right)+\frac{\partial}{\partial y}\left(\varepsilon_{y} \frac{\partial \bar{C}}{\partial y}\right)+\frac{\partial}{\partial z}\left(\varepsilon_{z} \frac{\partial \bar{C}}{\partial z}\right)+S .
$$

Utilizando as hipóteses simplificadoras encontradas em Bedmar (1972) e Barros (2004) na equação 
(4), obtém-se a equação unidimensional:

$$
\frac{\partial \bar{C}}{\partial t}+\bar{U} \frac{\partial \bar{C}}{\partial x}=\frac{\partial}{\partial x}\left(\varepsilon_{x} \frac{\partial \bar{C}}{\partial x}\right)+S
$$

Considerando que não há sumidouros ou processos físico-químicos com a concentração estudada, considera-se o termo fonte como uma função nula; além disso, o coeficiente de dispersão longitudinal ao longo do eixo $x$ é tomado constante neste estudo:

$$
S=0 ; \quad \varepsilon_{x}=D_{l},
$$

onde $D_{l}$ é o coeficiente de dispersão longitudinal constante $\left(\mathrm{m}^{2} / \mathrm{s}\right)$. Aplicando as simplificações (6) na equação (5),

$$
\frac{\partial C}{\partial t}+U \frac{\partial C}{\partial x}=D_{l} \frac{\partial^{2} C}{\partial x^{2}}, \quad t \geq 0
$$
tem-se a equação de dispersão de um poluente na forma unidimensional:

onde, neste caso, $C=C(x, t)$ representa a concentração média do poluente $\left(\mathrm{mg} / \mathrm{m}^{3}\right)$ e $U$ representa a velocidade média de escoamento $(\mathrm{m} / \mathrm{s})$ (as barras superiores foram retiradas por questão de simplificação nas notações).

Sendo:

$$
\begin{gathered}
\frac{\partial C}{\partial t} \text { - Representa a variação temporal da concentração; } \\
U \frac{\partial C}{\partial x} \text { - É o termo que representa o transporte advectivo e } \\
D_{l} \frac{\partial^{2} C}{\partial x^{2}} \text {-É o termo que representa o transporte difusivo. }
\end{gathered}
$$

Além disso, em problemas de dispersão de poluentes em rios, quando o poluente é despejado no corpo hídrico instantaneamente, considera-se que a equação (7) está sujeita à condição inicial:

$$
C(x, t=0)=\frac{M}{A} \delta(x)
$$

onde $M$ é a massa de traçador injetada $(m g), A$ é a área da seção molhada $\left(m^{2}\right)$, e $\delta(x)$ é a função Delta de Dirac.

$$
C(x= \pm \infty, t)=0 \quad t>0 .
$$

Para as condições de contorno, tem-se que:

No presente trabalho, iremos propor separadamente os modelos difusivo e advectivo para a dispersão de poluentes.

\section{Modelo Difusivo}

Nesta seção, será considerado o modelo que descreve a difusão turbulenta do poluente no corpo hídrico, não havendo advecção (não há velocidade de escoamento do rio, e, portanto $U=0$ ). Diante disso, a equação (7) se torna:

$\frac{\partial C}{\partial t}=D_{l} \frac{\partial^{2} C}{\partial x^{2}}$,

Para resolver a equação (10) sujeita às condições (8) e (9), serão utilizados os métodos GILTT e Taylor. Como o método GILTT não se aplica para domínio infinito, adota-se um domínio simétrico de $0<x<$ $L_{x}$, onde $L_{x}$ é o comprimento fictício do rio (suficientemente grande).

\section{Método GILTT}

Para resolver a equação (10), é preciso primeiramente resolver o problema auxiliar de SturmLiouville associado à equação:

$$
\psi_{n}^{\prime \prime}(x)+\lambda_{n}^{2} \psi_{n}(x)=0, \quad 0<x<L_{x}
$$




$$
\psi_{n}\left(L_{x}\right)=0, \quad \psi_{n}^{\prime}(0)=0,
$$

Que possui como resultado as autofunções

$\psi_{n}(x)=\cos \left(\lambda_{n} x\right)$

$$
\text { onde } \lambda_{n}=\left(n \pi-\frac{\pi}{2}\right) \frac{1}{L_{x}}, \quad n \in \mathbb{Z} \text { são os autovalores associados às autofunções (13). }
$$

Agora, expandindo a concentração em termos das autofunções do problema de Sturm-Liouville,

da seguinte forma:

$C(x, t)=\lim _{K \rightarrow \infty} \sum_{n=0}^{K} \overline{C_{n}}(t) \cdot \psi_{n}(x) \approx \sum_{n=0}^{N} \overline{C_{n}}(t) \cdot \psi_{n}(x)$,

onde $\overline{C_{n}}(t)$ são coeficientes a serem determinados e $N$ é o índice do somatório (suficientemente grande), no qual a soma (14) converge, deve-se substituir a equação (14) na Equação Diferencial Parcial (EDP) (10):

$\frac{\partial C}{\partial t}=D_{l} \frac{\partial^{2} C}{\partial x^{2}} \Rightarrow \frac{\partial}{\partial t}\left(\sum_{n=0}^{N} \overline{C_{n}}(t) \cdot \psi_{n}(x)\right)=D_{l} \frac{\partial^{2}}{\partial x^{2}}\left(\sum_{n=0}^{N} \overline{C_{n}}(t) \cdot \psi_{n}(x)\right) \Rightarrow$

$\Rightarrow \sum_{n=0}^{N} \overline{C_{n}{ }^{\prime}}(t) \cdot \psi_{n}(x)-\sum_{n=0}^{N} \overline{C_{n}}(t) \cdot D_{l} \cdot \psi_{n}^{\prime \prime}(x)=0$.

Aplicando o operador:

$\int_{0}^{L_{x}}(\cdot) \psi_{m}(x) d x$

em ambos os lados da equação (15), obtemos:

$\sum_{n=0}^{N} \overline{C_{n}{ }^{\prime}}(t) \cdot \int_{0}^{L_{x}} \psi_{n}(x) \psi_{m}(x) d x-\sum_{n=0}^{N} \overline{C_{n}}(t) \cdot \int_{0}^{L_{x}} D_{l} \psi_{n}^{\prime \prime}(x) \psi_{m}(x) d x=0$.

Sendo $b_{n, m}=\int_{0}^{L_{x}} \psi_{n}(x) \psi_{m}(x) d x$, será calculado seu valor:

$b_{n, m}=\int_{0}^{L_{x}} \cos \left(\lambda_{n} x\right) \cos \left(\lambda_{m} x\right) d x=\int_{0}^{L_{x}} \cos \left(\left(n \pi-\frac{\pi}{2}\right) \frac{1}{L_{x}} x\right) \cos \left(\left(m \pi-\frac{\pi}{2}\right) \frac{1}{L_{x}} x\right) d x=$

$=\int_{0}^{L_{x}} \frac{1}{2}\left[\cos \left((n+m-1) \pi \frac{x}{L_{x}}\right)+\cos \left((n-m) \pi \frac{x}{L_{x}}\right)\right] d x=$

$=\left.\frac{1}{2}[\underbrace{\frac{L_{x}}{(n+m-1) \pi} \sin \left((n+m-1) \pi \frac{X}{L_{x}}\right)}_{n \neq-m+1}+\underbrace{\frac{L_{x}}{(n-m) \pi} \sin \left((n-m) \pi \frac{X}{L_{x}}\right)}_{n \neq m}]\right|_{x=0} ^{L_{x}}$

$=0$.

Agora, se $n=m$, então:

$b_{n, m}=\int_{0}^{L_{x}} \cos \left(\left(n \pi-\frac{\pi}{2}\right) \frac{1}{L_{x}} x\right) \cos \left(\left(n \pi-\frac{\pi}{2}\right) \frac{1}{L_{x}} x\right) d x=\int_{0}^{L_{x}} \cos ^{2}\left(\left(n \pi-\frac{\pi}{2}\right) \frac{1}{L_{x}} x\right) d x=\frac{L_{x}}{2}$.

Logo, pelas equações (18) e (19), conclui-se que:

$b_{n, m}=\left\{\begin{array}{c}\frac{L_{x}}{2}, \text { se } m=n \geq 0 \\ 0, \text { se } m \neq n .\end{array}\right.$

Agora, reescrevendo a equação (17) na forma matricial:

$B \cdot Y^{\prime}(t)=E \cdot Y(t)$, 
Onde:

$$
\begin{aligned}
& Y(t)=\left\{\overline{C_{n}}(t)\right\} ; \\
& B=\left\{b_{n, m}\right\} ;
\end{aligned}
$$

$E=\left\{e_{n, m}\right\}$, onde $e_{n, m}=\int_{0}^{L_{x}} D_{l} \psi_{n}^{\prime \prime}(x) \psi_{m}(x) d x$.

Ou ainda, escrevendo a equação (21) como:

$Y^{\prime}(t)+F \cdot Y(t)=0$,

onde $F=-B^{-1} \cdot E$. A equação (22) está sujeita à condição inicial:

$Y(0)=\left\{\overline{C_{n}}(0)\right\}$.

Pela equação (14), expandindo também a condição inicial (23) e igualando à equação (8):

$C(x, 0)=\frac{M}{A} \delta(x) \Rightarrow C(x, 0)=\sum_{n=0}^{N} \overline{C_{n}}(0) \cdot \psi_{n}(x)=\frac{M}{A} \delta(x)$.

De maneira análoga, aplica-se o operador $\int_{0}^{L_{x}}(\cdot) \psi_{m}(x) d x$ na equação (24) e obtém-se:

$\sum_{n=0}^{N} \overline{C_{n}}(0) \cdot \int_{0}^{L_{x}} \psi_{n}(x) \psi_{m}(x) d x=\frac{M}{A} \int_{0}^{L_{x}} \delta(x) \psi_{m}(x) d x$.

Calculando as integrais da equação (25), obtêm-se:

$\overline{C_{n}}(0) \frac{L_{x}}{2}=\frac{M}{A} \Rightarrow \overline{C_{n}}(0)=\frac{2 M}{A L_{x}}$

Portanto, pela equação (26), a condição inicial (24) é reescrita na forma matricial como:

$Y(0)=\left\{\frac{2 M}{A L_{x}}\right\}$.

Finalmente, resolve-se a equação (22), aplicando a Transformada de Laplace em ambos os lados:

$$
\begin{aligned}
\mathcal{L}\left\{Y^{\prime}(t)+F \cdot Y(t), t \rightarrow s\right\} & =\mathcal{L}\{0, t \rightarrow s\} \Rightarrow s \cdot \mathcal{L}\{Y(t), t \rightarrow s\}-Y(0)+F \cdot \mathcal{L}\{Y(t), t \rightarrow s\}=0 \\
& \Rightarrow \\
& \Rightarrow s \cdot \mathcal{L}\{Y(t), t \rightarrow s\}+F \cdot \mathcal{L}\{Y(t), t \rightarrow s\} \\
& =Y(0) .
\end{aligned}
$$

Assumindo que $F$ seja diagonalizável, $F$ pode ser escrita da forma $F=X \cdot D \cdot X^{-1}$

onde $D$ é uma matriz diagonal cujos elementos são os autovalores de $F, X$ é uma matriz cujas colunas são os autovetores linearmente independentes de $F$, e $X^{-1}$ é sua inversa. Substituindo a equação (29) na equação (28): $s \cdot \mathcal{L}\{Y(t), t \rightarrow s\}+X \cdot D \cdot X^{-1} \cdot \mathcal{L}\{Y(t), t \rightarrow s\}=Y(0) \Rightarrow\left(s \cdot X \cdot X^{-1}+X \cdot D \cdot X^{-1}\right) \cdot \mathcal{L}\{Y(t), t \rightarrow s\}$

$$
\begin{aligned}
& =Y(0) \Rightarrow \\
& \quad \Rightarrow X \cdot(s \cdot I+D) \cdot X^{-1} \cdot \mathcal{L}\{Y(t), t \rightarrow s\}=Y(0) \Rightarrow \mathcal{L}\{Y(t), t \rightarrow s\} \\
& =X \cdot(s \cdot I+D)^{-1} \cdot X^{-1} \cdot Y(0) .
\end{aligned}
$$

Aplicando a Transformada inversa de Laplace, denotada por $\mathcal{L}^{-1}$, na equação (30):

$$
Y(t)=\mathcal{L}^{-1}\left\{X \cdot(s \cdot I+D)^{-1} \cdot X^{-1} \cdot Y(0), s \rightarrow t\right\}=X \cdot \mathcal{L}^{-1}\left\{(s \cdot I+D)^{-1}, s \rightarrow t\right\} \cdot X^{-1} \cdot Y(0) .
$$

Agora, será calculado $\mathcal{L}^{-1}\{(s \cdot I+D), s \rightarrow t\}$, da equação (31), denotando por $d_{0}, d_{1}, \ldots, d_{N}$ os autovalores da matriz $F$ : 


$$
\begin{gathered}
s \cdot I+D=\left(\begin{array}{ccc}
s+d_{0} & \cdots & 0 \\
\vdots & \ddots & \vdots \\
0 & \cdots & s+d_{N}
\end{array}\right) \Rightarrow(s \cdot I+D)^{-1}=\left(\begin{array}{ccc}
\frac{1}{s+d_{0}} & \cdots & 0 \\
\vdots & \ddots & \vdots \\
0 & \cdots & \frac{1}{s+d_{N}}
\end{array}\right) \\
\Rightarrow \\
\Rightarrow \mathcal{L}^{-1}\{(s \cdot I+D)\}=\left(\begin{array}{ccc}
\mathcal{L}^{-1}\left\{\frac{1}{s+d_{0}}, s \rightarrow t\right\} & \cdots & \mathcal{L}^{-1}\{0, s \rightarrow t\} \\
\vdots & \ddots & \vdots \\
\mathcal{L}^{-1}\{0, s \rightarrow t\} & \cdots & \mathcal{L}^{-1}\left\{\frac{1}{s+d_{N}}, s \rightarrow t\right\}
\end{array}\right)=\left(\begin{array}{ccc}
e^{-d_{0} t} & \cdots & 0 \\
\vdots & \ddots & \vdots \\
0 & \cdots & e^{-d_{N} t}
\end{array}\right) \\
=G(t) . \quad(32)
\end{gathered}
$$

Portanto, com a matriz $G(t)$ definida na equação (32), conclui-se que a solução para a equação (22) é:

$$
Y(t)=X \cdot G(t) \cdot X^{-1} \cdot Y(0),
$$

cujo resultado será o vetor coluna de elementos $\left\{\overline{C_{n}}(t)\right\}$.

Portanto, a solução da equação de difusão unidimensional dependente do tempo pelo método GILTT é representada pela expressão:

$$
C(x, t) \approx \sum_{n=0}^{N} \overline{C_{n}}(t) \cdot \psi_{n}(x),
$$

onde $\overline{C_{n}}(t)$ é definida na equação (33) e $\psi_{n}(x)$ é definido na equação (13).

\section{Método de Taylor}

Como mostrado detalhadamente em Socolofsky et al. (2002), para a resolução da equação (10) pelo método de Taylor, será considerada a solução da forma:

$$
C(x, t)=\frac{M}{A \sqrt{D_{l} t}} f_{1}\left(\frac{x}{\sqrt{D_{l} t}}\right)
$$

onde $f_{1}$ é uma função a ser determinada. Substituindo a equação (35) na EDP (10), a fim de tornála em uma EDO:

$$
\begin{gathered}
\frac{\partial C}{\partial t}=D_{l} \frac{\partial^{2} C}{\partial x^{2}} \Rightarrow \frac{\partial}{\partial t}\left[\frac{M}{A \sqrt{D_{l} t}} f_{1}\left(\frac{x}{\sqrt{D_{l} t}}\right)\right]=D_{l} \frac{\partial^{2}}{\partial x^{2}}\left[\frac{M}{A \sqrt{D_{l} t}} f_{1}\left(\frac{x}{\sqrt{D_{l} t}}\right)\right] \Rightarrow \\
\Rightarrow \frac{-1}{2 \sqrt{t^{3}}} f_{1}\left(\frac{x}{\sqrt{D_{l} t}}\right)+\frac{1}{\sqrt{t}} \frac{\partial}{\partial t}\left[f_{1}\left(\frac{x}{\sqrt{D_{l} t}}\right)\right]=D_{l} \frac{1}{\sqrt{t}} \frac{\partial^{2}}{\partial x^{2}}\left[f_{1}\left(\frac{x}{\sqrt{D_{l} t}}\right)\right] .
\end{gathered}
$$

Nomeando a variável adimensional como $\beta=\frac{x}{\sqrt{D_{l} t^{\prime}}}$, de forma que $\frac{\partial \beta}{\partial x}=\frac{1}{\sqrt{D_{l} t}} \mathrm{e} \frac{\partial \beta}{\partial t}=\frac{-x}{2 \sqrt{D_{l} t^{3}}}$, aplicando essas expressões na equação (36), tem-se:

$$
\begin{gathered}
\frac{-1}{2 \sqrt{t^{3}}} f_{1}(\beta)+\frac{1}{\sqrt{t}} f^{\prime}{ }_{1}(\beta) \frac{\partial \beta}{\partial t}=D_{l} \frac{1}{\sqrt{t}} \frac{\partial}{\partial x}\left[f^{\prime}{ }_{1}(\beta) \frac{\partial \beta}{\partial x}\right] \Rightarrow \\
\Rightarrow \frac{-1}{2 t} f_{1}(\beta)+{f^{\prime}}^{\prime}(\beta)\left(\frac{-x}{2 \sqrt{D_{l} t^{3}}}\right)=\left(\frac{D_{l}}{\sqrt{D_{l} t}}\right) f^{\prime \prime}{ }_{1}(\beta) \frac{\partial \beta}{\partial x} \Rightarrow
\end{gathered}
$$




$$
\begin{gathered}
\Rightarrow \frac{-1}{2} f_{1}(\beta)-\frac{1}{2} \underbrace{\left(\frac{x}{\sqrt{t D_{l}}}\right)}_{=\beta} f^{\prime}{ }_{1}(\beta)=f^{\prime \prime}{ }_{1}(\beta) \Rightarrow \\
\Rightarrow f_{1}(\beta)+\beta{f^{\prime}}_{1}(\beta)=-2 f^{\prime \prime}{ }_{1}(\beta) \Rightarrow \frac{d}{d \beta}\left[\beta f_{1}(\beta)\right]=-2 f^{\prime \prime}{ }_{1}(\beta) \Rightarrow \\
\Rightarrow \int\left(\frac{d}{d \beta}\left[\beta f_{1}(\beta)\right]\right) d \beta=\int\left(-2 f^{\prime \prime}{ }_{1}(\beta)\right) d \beta \Rightarrow \beta f_{1}(\beta)=-2 f^{\prime}{ }_{1}(\beta)+K_{1} .
\end{gathered}
$$

Assumindo que $K_{1}=0$ na equação (37), conclui-se que:

$$
\beta f_{1}(\beta)=-2 f^{\prime}{ }_{1}(\beta) \Rightarrow f_{1}(\beta)= \pm e^{-\frac{\beta^{2}}{4}} e^{K_{2}}=K e^{-\frac{\beta^{2}}{4}} \text {. }
$$

Portanto, como foi suposto que a solução da equação (10) era da forma $C(x, t)=\frac{M}{A_{\sqrt{D_{l} t}}} f_{1}\left(\frac{x}{\sqrt{D_{l} t}}\right)$, conclui-se que:

$$
C(x, t)=\frac{M}{A \sqrt{D_{l} t}} f_{1}\left(\frac{x}{\sqrt{D_{l} t}}\right)=K \frac{M}{A \sqrt{D_{l} t}} e^{-\frac{\left(\frac{x}{\sqrt{D_{l} t}}\right)^{2}}{4}} \Rightarrow C(x, t)=K \frac{M}{A \sqrt{D_{l} t}} e^{-\frac{x^{2}}{4 D_{l} t}} .
$$

Para determinar o valor da constante $K$ utiliza-se a condição inicial (8), porém como a função encontrada em (39) não está definida em $t=0$, utiliza-se a condição de continuidade:

$$
\lim _{t \rightarrow 0} C(x, t)=C(x, 0) .
$$

Portanto, igualando as equações (8) e (40), tem-se:

$$
C(x, t=0)=\frac{M}{A} \delta(x) \Rightarrow \lim _{t \rightarrow 0} K \frac{M}{A \sqrt{D_{l} t}} e^{-\frac{x^{2}}{4 D_{l} t}}=\frac{M}{A} \delta(x),
$$

aplicando a Transformada de Fourier (denotada por $\mathcal{F}$ ) em ambos lados da equação (41) e utilizando suas propriedades:

$$
\lim _{t \rightarrow 0} K \frac{M}{A \sqrt{D_{l} t}} \mathcal{F}\left\{e^{-\frac{x^{2}}{4 D_{l} t}}, x \rightarrow \omega\right\}=\frac{M}{A} \cdot 1 .
$$

Obtendo assim:

$$
\begin{aligned}
& \lim _{t \rightarrow 0} K \frac{M}{A \sqrt{D_{l} t}}\left(\sqrt{4 D_{l} t \pi} e^{-\omega^{2} D_{l} t}\right)=\frac{M}{A} \Rightarrow \\
& \Rightarrow \lim _{\mathrm{t} \rightarrow 0} 2 K \sqrt{\pi} e^{-\omega^{2} D_{l} t}=1 \Rightarrow 2 K \sqrt{\pi} \cdot 1=1 \Rightarrow \mathrm{K}=\frac{1}{2 \sqrt{\pi}} .
\end{aligned}
$$

Encontrado o valor de $K$ na equação (43), substitui-se em (39), e tem-se a solução do modelo difusivo pelo método de Taylor:

$$
C(x, t)=\frac{M}{2 A \sqrt{\pi D_{l} t}} e^{-\frac{x^{2}}{4 D_{l} t}}
$$

\section{Modelo Advectivo}

O transporte puramente advectivo provoca o deslocamento de partículas de soluto com a velocidadereal $(U)$ do fluido na direção do escoamento, sem considerar a difusão molecular do soluto, ou seja, $D_{l}=0$. Diante disso, a equação (7) se torna: 
$\frac{\partial C}{\partial t}+U \frac{\partial C}{\partial x}=0$

Para resolver a equação (45) sujeita às condições (8) e (9), utiliza-se o método de Separação de Variáveis com Transformada de Laplace.

\section{Método de Separação de Variáveis com Transformada de Laplace}

Começamos a resolução da equação (45) pela Separação de Variáveis. Supondo que a função $C(x, t)$ seja da forma:

$$
C(x, t)=X(x) \Gamma(t),
$$

e substituindo a equação (46) na equação (45):

$\frac{\partial[X(x) \Gamma(t)]}{\partial t}+U \frac{\partial[X(x) \Gamma(t)]}{\partial x}=0$.

Aplicando o operador diferencial na solução proposta, segue:

$$
X(x) \Gamma^{\prime}(t)+U X^{\prime}(x) \Gamma(t)=0,
$$

Obtendo como resultado:

$\frac{1}{U} \frac{\Gamma^{\prime}(t)}{\Gamma(t)}=-\frac{X^{\prime}(x)}{X(x)}$.

Como é de se notar, a expressão à esquerda da equação (49) depende somente de $t$ e a expressão à direita depende somente de $x$. Logo, para que essa igualdade seja válida, é preciso que ambos os lados da equação sejam iguais à mesma constante. Denotando essa constante de separação $\operatorname{como} \alpha$, tem-se:

$$
\frac{1}{U} \frac{\Gamma^{\prime}(t)}{\Gamma(t)}=-\frac{X^{\prime}(x)}{X(x)}=\alpha .
$$

Ou seja, são obtidas as duas EDO's:

$$
\frac{1}{U} \frac{\Gamma^{\prime}(t)}{\Gamma(t)}=\alpha
$$

$\mathrm{E}$

$$
-\frac{X^{\prime}(x)}{X(x)}=\alpha \text {. }
$$

Considerando as equações (51) e (52), obtêm-se como soluções:

$$
\Gamma(t)=A e^{\alpha U t} \text { e } \quad X(x)=B e^{-\alpha x} \text {. }
$$

Portanto, a solução da equação (45) será:

$$
C(x, t)=B e^{-\alpha x} A e^{\alpha U t}=K e^{-\alpha x} e^{\alpha U t} \text {. }
$$

Além disso, pela condição de contorno (9), tem-se que:

$$
\lim _{x \rightarrow \infty} C(x, t)=0 \text {, }
$$

ou seja,

$\lim _{x \rightarrow \infty} K e^{-\alpha x} e^{\alpha U t}=0 \Rightarrow K e^{\alpha U t} \lim _{x \rightarrow \infty} e^{-\alpha x}=0$.

Como pode-se ver, a constante de separação $(\alpha)$ deve ser um número real positivo. Com isso, reescreve-se a solução final como: 
$C(x, t)=\int_{0}^{\infty} D(\alpha) e^{\alpha U t} e^{-\alpha x} d \alpha$.

Para determinar o coeficiente $D(\alpha)$, será utilizada a condição inicial (8):

$C(x, 0)=\frac{M}{A} \delta(x) \Rightarrow \int_{0}^{\infty} D(\alpha) e^{-\alpha x} d \alpha=\frac{M}{A} \delta(x)$.

Observa-se que o lado esquerdo da equação (58) é, por definição, a Transformada de Laplace da função $D(\alpha)$, transformando $\alpha$ em $x$. Desta forma, será aplicada a Transformada inversa de Laplace em ambos os lados da equação:

$$
\mathcal{L}^{-1}\{\mathcal{L}\{D(\alpha), \alpha \rightarrow x\}, x \rightarrow \alpha\}=\mathcal{L}^{-1}\left\{\frac{M}{A} \delta(x), x \rightarrow \alpha\right\}
$$

Assim, tem-se:

$$
D(\alpha)=\mathcal{L}^{-1}\left\{\frac{M}{A} \delta(x), x \rightarrow \alpha\right\} \text {. }
$$

Substituindo na equação (57), 1obtém-se:

$$
C(x, t)=\int_{0}^{\infty} \mathcal{L}^{-1}\left\{\frac{M}{A} \delta(x), x \rightarrow \alpha\right\} e^{\alpha U t} e^{-\alpha x} d \alpha=\frac{M}{A} \delta(x+U t) .
$$

Portanto a equação (61) é a solução para o problema do transporte advectivo de poluentes.

\section{RESULTADOS E DISCUSSÃO}

Tendo em vista as soluções para o modelo difusivo para a dispersão de poluentes em rios dadas pelas equações (34) e (44), e a solução para o modelo advectivo, dada pela equação (61), foi simulada a dispersão de poluentes no rio Paraibuna, usando os parâmetros hidráulicos obtidos no trabalho de Soares et al. (2010): a massa injetada do traçador $M=500.000 \mathrm{mg}$; a área transversal média do rio $A=30,138 \mathrm{~m}^{2}$; coeficiente de dispersão longitudinal $D_{l}=6,2 \mathrm{~m}^{2} / \mathrm{s}$ e velocidade $U=0,52 \mathrm{~m} / \mathrm{s}$. Os cálculos apresentados nas seções anteriores foram programados e executados no software Julia v.1.4.2, e os gráficos com os dados obtidos foram plotados no software Octavev.5.1.0.

A Figura 1 mostra a simulação feita acerca do modelo difusivo, em um gráfico da concentração do traçador (em $p p b)$ em função da posição a partir da fonte $(\mathrm{em} \mathrm{km}$ ), tendo fixo três momentos distintos e suficientemente espaçados (1/2 dia, 1 dia e 5 dias após a injeção de traçador).

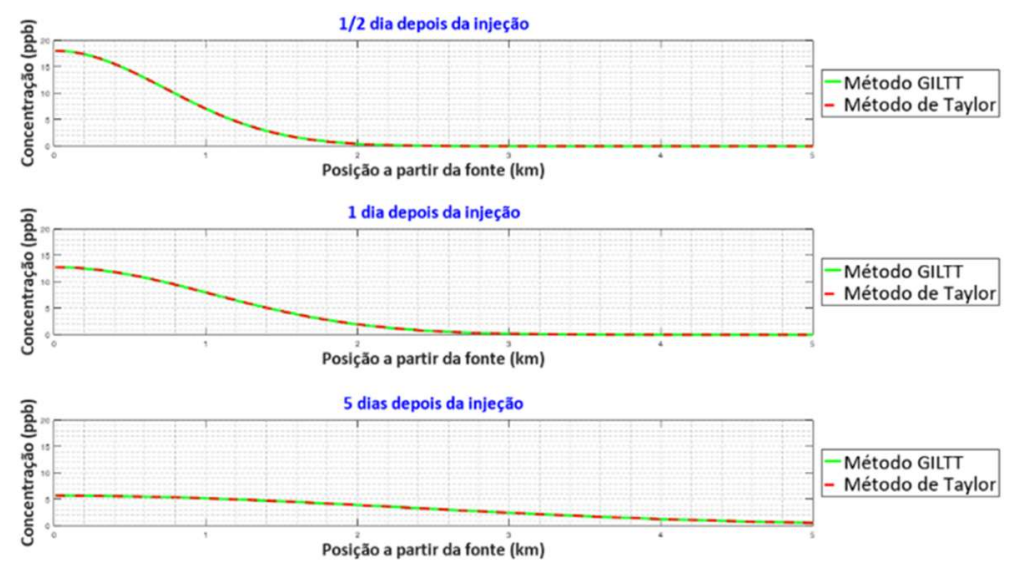

Figura 1: Simulação do modelo difusivo pelos métodos de Taylor e GILTT para os instantes 1⁄2 dia, 1 dia e 5 dias. 
Como pode-se observar na Figura 1, a concentração máxima está situada próximo ao ponto de injeção de traçadores, dispersando-se vagarosamente para o restante do rio, no processo de homogeneização da quantidade de traçador ao longo de todo o trecho analisado. Esse processo se dá lentamente pois, como observado do modelo difusivo, consideramos a velocidade do rio como nula, e examina-se apenas a atividade de dispersão turbulenta das partículas de poluente inseridas norio.

Além disso, comparando a simulação apresentada na Figura 1 com os dados das curvas de passagem do traçador obtidos na campanha no rio Paraibuna de Soares et al. (2010), encontrados nas Figuras 3b, 3c e $3 d$, é possível analisar a diferença na quantidade de traçador dissipada pelo rio no processo difusivoadvectivo; nos dados observados, após aproximadamente 10 horas da injeção, a concentração apresenta um pico na posição próxima de $20 \mathrm{~km}$, enquanto nos dados simulados para o modelo puramente difusivo observa-se que o pico de concentração após 12 horas da injeção será de localizado a menos de $1 \mathrm{~km}$ do ponto de injeção. A Figura 2 apresenta a concentração de traçador (em $p p b$ ) em função do tempo a partir da injeção, em três posições fixas a partir da fonte $(100 \mathrm{~m}, 500 \mathrm{~m}$ e $1 \mathrm{~km})$.

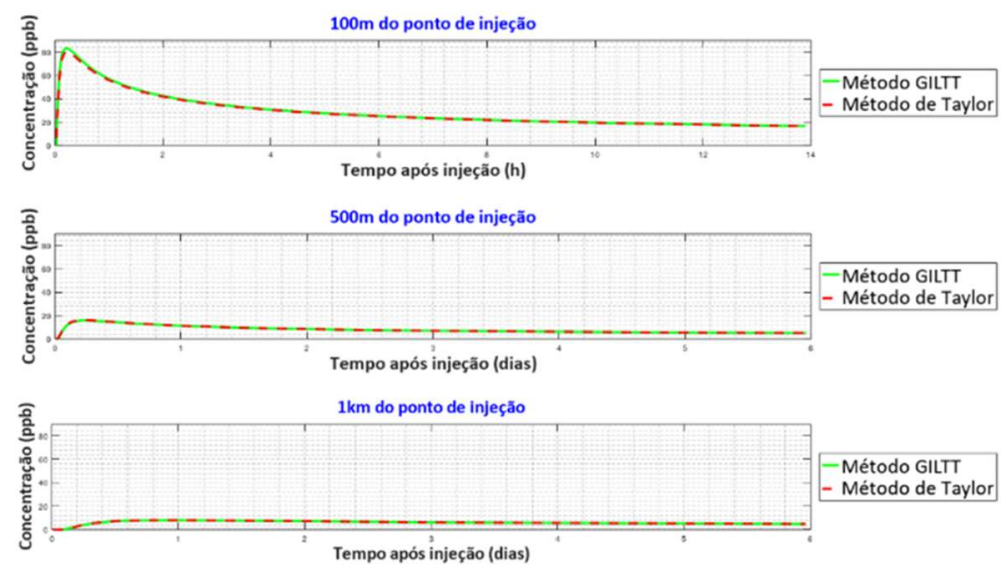

Figura 2: Simulação do modelo difusivo pelos métodos de Taylor e GILTT para as posições $100 \mathrm{~m}, 500 \mathrm{~m}$ e $1 \mathrm{~km}$.

Analogamente à Figura 1, notamos que, na Figura 2, o traçador se concentra em maior quantidade em locais próximos à fonte (como em $100 \mathrm{~m}$ ), e leva um tempo considerável para a diluição para o restante do rio, o que pode ser notado principalmente em pontos localizados distantes da fonte (como em $1 \mathrm{~km}$ ). De fato, analisando a posição $x=100 \mathrm{~m}$, obtem-se que em $t=0,2 \mathrm{~h}$ a concentração é de $C=80 p p b$, e neste mesmo ponto a concentração diminui $75 \%$ somente após, aproximadamente, $9,44 h$ da injeção de traçador. Enquanto isso, analisando a posição $x=1 \mathrm{~km}$, a concentração máxima se mantém, durante todo o processo, abaixo de $C=10 p p b$, cerca de $12,5 \%$ da concentração máxima em $x=100 \mathrm{~m}$.

Quanto ao modelo advectivo, foi feita a comparação entre os dados simulados e os dados observados na campanha de Soares et al. (2010). Na Figura 3a, foram plotados os dados simulados, e nas Figuras 3b, 3c e $3 d$ estão representados os dados reais para o transporte da nuvem de traçador (em $p p b$ ) em função do tempo (em $h$ ), para três posições fixas $(7,6 \mathrm{~km}, 16 \mathrm{~km}$ e $20 \mathrm{~km})$.

Em análise à Figura 3a, é possível notar que a nuvem de traçador permanece intacta durante o processo de advecção, ou seja, a nuvem de traçador desloca-se em um bloco que não se difunde para os seus arredores, como discutido na seção do modelo advectivo. Neste caso, a quantidade de traçador observada 
no gráfico simulado da Figura 3aé sempre igual à condição inicial $\frac{M}{A}$. Além disso, comparado aos dados reais nas Figuras 3b, 3c e 3d, pode-se ver que o pico de traçador em cada posto de análise ocorre em momentos aproximados, o que poderia ser esperado, pelo fato da advecção ser o processo dominante na dispersão de poluentes em meios hídricos.
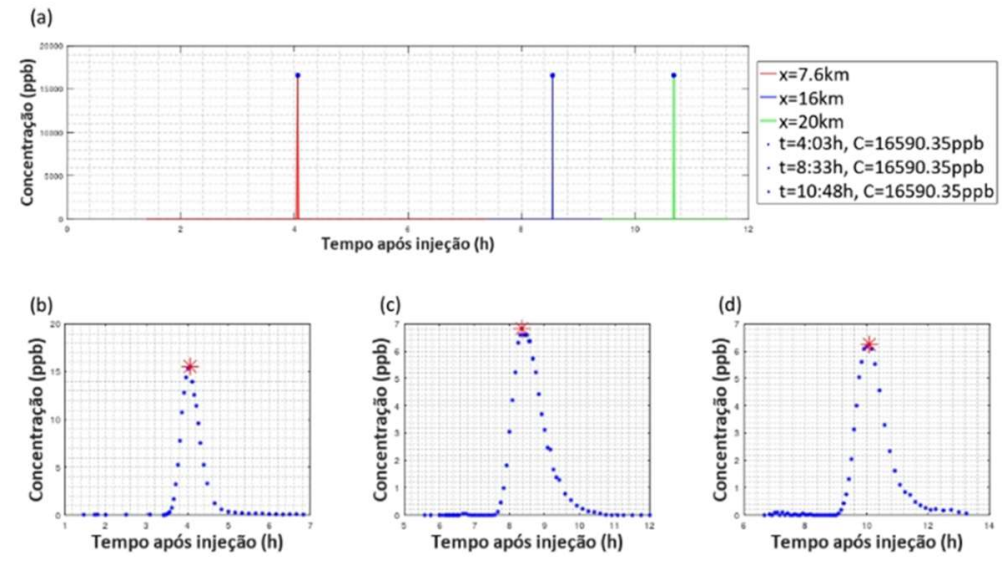

- Dados Observados em: (b) $x=7,6 \mathrm{~km} ; \quad$ (c) $x=16 \mathrm{~km} ; \quad$ (d) $x=20 \mathrm{~km}$

- Ponto de maior concentração: (b) $(t=4: 03 \mathrm{~h}, \mathrm{C}=15.55 \mathrm{ppb}) ; \quad$ (c) $(t=8: 21 \mathrm{~h}, \mathrm{C}=6.83 \mathrm{ppb}) ; \quad(d)(t=10: 04 \mathrm{~h}, \mathrm{C}=6.25 \mathrm{ppb})$.

Figura 3: Simulação do modelo Advectivo por Separação de Variáveis e Transformada de Laplace, em comparação com os dados da natureza medidos em experimento nas posições $7.6 \mathrm{~km}, 16 \mathrm{~km}$ e $20 \mathrm{~km}$.

\section{CONCLUSÕES}

A preservação dos meios hídricos é de extrema importância para a manutenção do ecossistema. Logo, é fundamental que o ser humano crie métodos visando a prevenção de incidentes danosos aos corpos hídricos, e a modelagem matemática vem exercendo com sucesso o trabalho de previsão e simulação de acidentes envolvendo a poluição de rios, mares e oceanos.

No presente trabalho, foi feita a comparação entre os modelos difusivo e advectivo para a dispersão de poluentes no rio Paraibuna. Foram apresentados métodos para a resolução de cada modelo e suas respectivas soluções analíticas para o caso unidimensional, e, a partir destes, foram apresentados gráficos de simulação para cada um desses modelos, bem como os dados reais observados.

A partir da análise realizada, verificou-se que ambos modelos são capazes de fornecer informações pertinentes quanto à dispersão de poluentes em corpos hídricos. Quando são analisados os dois casos separadamente, percebe-se que no modelo difusivo, o processo de dispersão ocorre lentamente, apresentando com eficácia a análise da dispersão turbulenta das partículas do poluente inserido no rio. Já o modelo advectivo apresenta o modo como a nuvem de traçador se desloca pelo leito do rio, e é eficaz para aproximar o momento de pico do poluente em cada posição.

O estudo realizado através dos modelos advectivo e difusivo mostraram a influência destes processos no problema físico, percebeu-se que o modelo advectivo tem uma influência maior no processo de dispersão do poluente. Este modelo é uma ótima escolha para determinação do instante (ou posição) dos pontos de maior concentração de poluente. Já o modelo difusivo o processo de dispersão é mais lento, contudo os resultados mostram a importância de levar em consideração este tipo de transporte nos modelos de 
dispersão. Desta forma, para uma análise mais condizente dos dados reais, é necessário o uso do modelo levando em consideração os dois processos de transporte de poluente, um modelo advectivo-difusivo.

\section{REFERÊNCIAS}

BARROS, F.. Modelos multidimensionais para dispersão de contaminantes em rios e canais: soluções híbridas por transformação integral. Dissertação (Mestrado em Engenharia Mecânica) - Universidade Federal do Rio de Janeiro, Rio de Janeiro, 2004.

BEDMAR, A. P.. Isótopos en hidrología. Madri: Alhambra, 1972.

BOYCE, W. E.; DIPRIMA, R. C.. Equações diferenciais elementares e problemas de valores de contorno. $8 \mathrm{ed}$. Rio de Janeiro: LTC, 2006.

BUSKE, D.; VILHENA, M. T.; SEGATTO, C. F.; QUADROS, R. S.. A General Analytical Solution of the Advection-Diffusion Equation for Fickian Closure. In: CONSTANDA, C.; HARRIS, P.. Integral Methods in Science and Engineering. Boston: Birkhäuser, 2011. p.25-34.

BUSKE, D.; VILHENA, M.; MOREIRA, D.; TIRABASSI, T. A.. General analytical solution of the transient two-dimensonal advection-diffusion equation with non-fickian closure in Cartesian geometry by the generalized integral transform technique. In: Integral methods in science and engineering: analytic methods. Boston: Birkhäuser, 2010, p.33-40.

BUSKE, D.; QUADROS, R. S.; OLIVEIRA, R. E.; WEYMAR, G. J.; HARTER, F. P.. Analytical solution for contaminant dispersion model in rivers and canals applying the method GILTT. International journal of development research, v.7, p.13857-13864, 2017.

ÇENGEL, Y. A.; CIMBALA, J. M.. Mecânica dos Fluídos: fundamentos e aplicações. São Paulo: AMGH Ltda., 2007.

CREMASCO, M. A.. Fundamentos de Transferência de Massa. 2 ed. São Paulo: Unicamp, 2009.

MACHADO, B. R.. Modelagem da dispersão de poluentes em rios e canais sob a perspectiva das abordagens GILTT e separação de variáveis. Dissertação (Mestrado em Ciências
Ambientais) - Universidade Federal de Pelotas, Pelotas, 2019.

MAIA, N. B.; MARTOS, H. L.; BARRELLA, W.. Indicadores ambientais: conceitos e aplicações. São Paulo: EDUC, 2001.

MOREIRA, D.; VILHENA, M.; BUSKE, D.; TIRABASSI, T.. The state-of-art of the GILTT method to simulate pollutant dispersion in the atmosphere. Atmospheric Research, v.92, p.1-17, 2009.

SOARES, J. H. P.; RIBEIRO, A. C. J.; GUEDES, H. A. S.. Avaliação da Capacidade de Transporte e Dispersão do Rio Paraibuna Utilizando Traçadores Fluorescentes. Trecho: Distrito Industrial a UHE Marmelos - Juiz de Fora/MG. Revista Brasileira de Recursos Hídricos, v.15, n.3, p.45-56, 2010.

SOARES, R.; MACHADO, W. T. V.; CAMPOS, D. V. B.; MONTEIRO, M. I. C.; FREIRE, A. S.; SANTELLI, R. E.. Avaliação da aplicabilidade de índices de poluição aquática: Estudo de caso no rio Paraibuna (Juiz de Fora, MG, Brasil). Revista Virtual de Química, Niterói, v.8, n.6, p.2105-2122, 2016.

SOCOLOFSKY, S. A.; JIRKA, G. H.. Environmental fluid mechanics part I: mass transfer and diffusion. $2 \mathrm{ed}$. Karlsruhe: Universität Karlsruhe, 2002.

TIRABASSI, T.; TIESI, A.; BUSKE, D.; VILHENA, M. T. M. D. Some characteristics of a plume from a point source based on analytical solution of the two-dimensional advctiondiffusion equation. Atmospheric Environment, v.43, p.22212227, 2009.

VILHENA, M. T.; BUSKE, D.; DEGRAZIA, G. A.; QUADROS, R. $S$.. An analytical model with temporal variable eddy diffusivity applied to contaminant dispersion in the atmospheric boundary layer. Physica A: Statistical Mechanics and its Applications, v.391, p.2576-2584, 2012.

WORTMANN, S.; VILHENA, M. T.; MOREIRA, D. M.; BUSKE, D.. A new analytical approach to simulate the pollutant dispersion in the PBL. Atmospheric Environment, v.39, p.2171-2178, 2005.

A CBPC - Companhia Brasileira de Produção Científica (CNPJ: 11.221.422/0001-03) detém os direitos materiais desta publicação. Os direitos referem-se à publicação do trabalho em qualquer parte do mundo, incluindo os direitos às renovações, expansões e disseminaç̃oes da contribuição, bem como outros direitos subsidiários. Todos os trabalhos publicados eletronicamente poderão posteriormente ser publicados em coletâneas impressas sob coordenação da Sustenere Publishing, da Companhia Brasileira de Produção Científica e seus parceiros autorizados. Os (as) autores (as) preservam os direitos autorais, mas não têm permissão para a publicação da contribuição em outro meio, impresso ou digital, em português ou em tradução. 